\title{
Patient perspectives of participating in the cardiac CopenHeartRFA rehabilitation program for patients treated with ablation for atrial fibrillation
}

This article was published in the following Dove Press journal: Journal of Multidisciplinary Healthcare

\author{
Signe S Risom ${ }^{1,2}$ \\ Johanne Lind ${ }^{2}$ \\ Pamela J McCabe ${ }^{3}$ \\ Selina Kikkenborg Berg',4,5 \\ 'The Heart Centre, Rigshospitalet, \\ Copenhagen University Hospital, \\ Copenhagen, Denmark; ${ }^{2}$ Department \\ of Nursing, Metropolitan University \\ College, Copenhagen, Denmark; \\ ${ }^{3}$ Mayo Clinic, Rochester, MN, USA; \\ ${ }^{4}$ Faculty of Health and Medical \\ Sciences, University of Copenhagen, \\ Copenhagen, Denmark; ${ }^{5}$ National \\ Institute of Public Health, University \\ of Southern Denmark, Copenhagen, \\ Denmark
}

Purpose: The CopenHeartRFA trial was designed and conducted to explore the effects of rehabilitation on patients treated with ablation for atrial fibrillation (AF). It included a cardiac rehabilitation program consisting of physical exercise and psychoeducational consultations over 6 months. As part of the evaluation of the CopenHeartRFA trial, both quantitative and qualitative data were collected. The intervention was delivered by a multidisciplinary team.

Aim: This study aimed to understand the experience of patients treated for AF with ablation who participated in the CopenHeartRFA cardiac rehabilitation program.

Methods: A qualitative descriptive design was used. A purposive sample of 10 patients who had completed the intervention consented to participate. The interviews were conducted faceto-face and were audio-recorded and transcribed. Qualitative content analyses were used to analyze the data. The interviews were analyzed individually by two researchers and themes were constructed and discussed.

Results: The sample included 10 participants, mean age 54.6 years. Four categories were identified and labeled: 1) strengthening belief in one's own physical capacity and survival; 2) still struggling with fear of AF symptoms; 3) a need for support to move on; and 4) regaining confidence in one's own mental strength with support from professionals.

Conclusion: The four themes describe a positive experience from participating in the rehabilitation program. The rehabilitation program promoted self-efficacy for engaging in physical activity and the benefit of a safe environment for processing the emotional responses and the value of the therapeutic relationship between the patient and the rehabilitation provider.

Keywords: atrial fibrillation, rehabilitation, qualitative research

\section{Introduction}

Annually, nearly five million people are diagnosed with atrial fibrillation (AF) worldwide $^{1}$ and the prevalence of AF is predicted to increase in the future. ${ }^{2}$ Patients with AF suffer increased mortality, severe stroke event rates, and heart failure. ${ }^{3}$ Consequently, treatment aims to reduce symptoms, avoid complications, and improve health-related quality of life. ${ }^{3,4}$ Catheter ablation is offered to symptomatic patients with a success rate of around $70 \% .{ }^{3}$ Patients' experience of health with AF has been described in a qualitative study. ${ }^{5}$ They try to decipher the meaning of their symptoms, feel uninformed and unsupported, and also feel emotionally distressed..$^{5}$

Favorable effects of cardiac rehabilitation for patients with ischemic heart disease and heart failure have been found. ${ }^{6}$ To examine whether those positive effects were transferable to patients treated with ablation for AF, the CopenHeartRFA trial, a
Correspondence: Signe S Risom

The Heart Centre, Rigshospitalet, Copenhagen University Hospital, Unit 2152, Blegdamsvej 9, 2100 Copenhagen, Denmark

Tel +4551632786

Fax +45 35457705

Email signe.stelling.risom@regionh.dk 
multidisciplinary comprehensive cardiac rehabilitation program consisting of physical exercise and psychoeducational consultations including 210 patients, was conducted. The design and methods used in the randomized CopenHeartRFA trial have previously been described. ${ }^{7}$ Primary results showed that compared with usual care, patients in the cardiac rehabilitation group improved in their physical capacity measured by $\mathrm{VO}_{2}$ peak at 4 months (24.3 vs $20.7 \mathrm{~mL} \mathrm{~kg}^{-1} \mathrm{~min}^{-1}$, $P=0.003)$. Perceived health measured by the Short Form-36 questionnaire showed no significant difference between groups at 6 months (53.8 vs 51.9 points, $P=0.20) .{ }^{8}$ As a part of the evaluation of the trial, both quantitative and qualitative measures were planned in a mixed method design. ${ }^{7,9}$ In this study, we report the results from the qualitative data that were collected after the quantitative findings.

The purpose of conducting the qualitative interviews was to provide an in-depth understanding of how the patients described their experiences in participating in the cardiac rehabilitation program. ${ }^{9}$ Understanding the experience of patients who participate in cardiac rehabilitation programs after ablation of AF adds knowledge about patient-centered outcomes. Patients' descriptions of barriers, challenges, and positive effects in the program will provide crucial knowledge to guide future research and implementation of cardiac rehabilitation for patients treated for AF.

\section{Methods}

A qualitative descriptive design was used.

\section{Setting and patients}

The trial was conducted at two Danish University hospitals. Patients treated with radiofrequency catheter ablation (RFA) for $A F$ were included. Inclusion criteria were $\geq 18$ years of age, Danish speaking patients. Exclusion criteria included those who were unable to understand trial instructions, pregnant or breastfeeding patients, with reduced ability to follow the planned program due to other physical illnesses, those who prior to RFA had been engaged in intense physical exercise at a competitive level, or were enrolled in other clinical trials.

A purposive sample of 10 patients who had completed the intervention, representing both sexes and types of AF (paroxysmal/persistent AF), was asked to participate. Ten patients represent $10 \%$ of the population in the cardiac rehabilitation group and were considered a large enough population for providing valid information on in-depth understanding how the patients described their experience in participating in the cardiac rehabilitation program and the analysis showed that information saturation was gained. ${ }^{10,11}$ We included patients of both sexes since sex differences have been found in rehabilitation outcomes. ${ }^{12}$ Patients with both types of AF were included since that can influence the way AF symptoms are experienced. ${ }^{3}$

The cardiac rehabilitation intervention has previously been described in detail in an earlier paper. ${ }^{7}$ Briefly, the intervention was carried out by a multidisciplinary team and consisted of a 12-week physical exercise program and psychoeducational consultations. The physical exercise program aimed at increasing physical capacity. The program was initiated at the hospital where the trial was conducted. After introduction to the program patients could opt for exercise training at the hospital where they were introduced to the program, their local hospital, or training at home. Therefore, patients could train in groups or alone. All patients followed a training diary and used a pulse-watch. Four psychoeducational consultations were inspired by Parse's Human Becoming Practice Methodologies ${ }^{13}$ and the overall aim was to offer emotional support, promote coping skills, and illness appraisal for participants to be able to respond properly to physical and psychological symptoms. A nurse was present in the process through discussions, silent immersion, and reflection. Education and information about the disease prepared the patient if symptoms would recur; these were tailored individually and only given when relevant to the patient. The Human Becoming Practice methodology was chosen to apply a holistic approach to the patient, focusing on the process where the patient was coping and transforming back to their everyday life. ${ }^{7}$

\section{Data collection}

At the end of the intervention, 10 participants were mailed a letter informing them about the interview and that they would receive a telephone call asking if they would like to participate in the interview regarding their experience with the cardiac rehabilitation program. Semi-structured interviews lasting around 30 minutes were conducted from February 2014 through March 2014. Examples of interview questions from the developed interview guide were: "How was your experience participating in the program?"; "What did it mean to you to participate in the exercise training and in the consultations with a nurse?"; "Can you give me some examples of what you discussed with the nurse?" The interviews were conducted face-to-face in a meeting room at the hospital and were audio-recorded. The first author (SSR) conducted the interviews, but was not involved in delivering the intervention and did not have contact with the participants prior to the interview. 


\section{Data analysis}

Audio recordings were transcribed verbatim. The transcriptions were compared with the audio recording to assure accuracy of the data. Qualitative content analysis ${ }^{10,14}$ was used to analyze the data. Naïve reading and coding were performed by two authors (SSR and JLR) independent of each other. NVivo version 11 software (QSR International, Melbourne, VIC, Australia) was used to aid in the sorting and coding of data. The interviews were analyzed step by step and line by line. Both authors coded the text into categories and subcategories that reflected common patterns and ideas. Then, the authors met and together the codes were grouped into categories or subcategories and after an additional abstraction the themes emerged. Subsequently, each category and theme were discussed. If any discrepancy occurred, a consensus was reached by discussion. The translation of the quotes from Danish to English was done by Signe S Risom after the analysis and after the quotes were chosen for the manuscript.

\section{Trustworthiness}

Several strategies were employed to assure trustworthiness of the findings. In the first phase of analysis, two authors analyzed the data independently of each other; one author (SSR) was involved in the trial but the other author (JLR) was not involved in the trial prior to data analysis. Therefore, JLR was able to naively interpret the results without knowledge of the trial events. This approach allowed for discussions and categorizations that might not have taken place if both authors had worked on the trial previously. This approach reduced bias, promoted broader interpretations, and stimulated discussions about discrepancies that produced a more thorough and complete analysis.

\section{Ethical approval}

The trial was approved by the local Ethics Committee (The National Committee on Health Research Ethics, number H-1-2011-135) and the Danish Data Protection Agency (reg number 2007-58-0015), registered at ClinicalTrials. gov (NCT01523145), and complied with the Declaration of Helsinki. Written informed consent was obtained from all participants before enrollment in the trial.

\section{Results}

The sample included 10 participants. The mean age was 54.6 years and most were living with a partner and had $>3$ years of education after high school level. Four patients had previous ablations and three patients presented with AF after ablation (Table 1). Four categories were identified and labeled (Table 2).
Table I Demographics and clinical characteristics $(\mathrm{N}=10)$

\begin{tabular}{|c|c|}
\hline Baseline characteristics & $\mathbf{N}(\%)$ \\
\hline \multicolumn{2}{|l|}{ Age in years } \\
\hline Mean (SD) & $54.6(11.6)$ \\
\hline Median (range, min-max) & $59.6(33-63)$ \\
\hline Male & $6(60)$ \\
\hline AF type - paroxysmal & $6(60)$ \\
\hline \multicolumn{2}{|l|}{ Living with partner } \\
\hline Yes & $8(80)$ \\
\hline No & $2(20)$ \\
\hline \multicolumn{2}{|c|}{ Further education (after high school level) } \\
\hline$\leq 3$ years & $4(40)$ \\
\hline$>3$ years & $6(60)$ \\
\hline \multicolumn{2}{|l|}{ Comorbidities } \\
\hline Hypertension & $4(40)$ \\
\hline Ischemic heart disease & $0(0)$ \\
\hline Diabetes & $0(0)$ \\
\hline \multicolumn{2}{|l|}{ Previous ablations } \\
\hline Yes & $4(40)$ \\
\hline No & $6(60)$ \\
\hline \multicolumn{2}{|l|}{ AF after ablation* } \\
\hline Yes & $3(30)$ \\
\hline No & $6(60)$ \\
\hline Do not know & $\mathrm{I}(\mathrm{I0})$ \\
\hline
\end{tabular}

Note: *Patients were asked if they had had AF at 4 months follow-up visit. Abbreviations: AF, Atrial fibrillation; SD, standard deviation.

\section{Strengthening belief in one's own physical capacity and survival}

Participants described how the physical training sessions reestablished their belief in survival. By participating in the training sessions, participants experienced both increased physical and mental capacity:

\section{So, after I got started on that training course and so, it has been giving me some mental strength, I'm in better shape and it has giving me something physical, right? I can handle more again, right. [Participant 5, male]}

The belief in survival was supported by the physiotherapists who motivated the participants and by the experience of feeling safe during the training sessions. To be introduced to the sessions and supervised by a physiotherapist felt motivating for the participants. That the sessions were structured and the training was performed wearing a pulse rate monitor was considered motivating as well.

[...] I have used it [the pulse rate monitor] as a kind of guideline to say okay now you should watch out that your pulses are not getting too high or too low, you need to get up here [the pulse rate monitor]. [Participant 9, male]

Participants were concerned with safety and found it safe to perform the training sessions with a physiotherapist who could 
Table 2 Participants experiences after partaking in the cardiac rehabilitation program

Theme
Strengthening belief in one's own
physical capacity and survival

Examples in participant's own words

Still struggling with fear of atrial fibrillation symptoms

So it's both the physical, but it also affects the psychological state that you get to do those things. [Particpant 5, male] But you also feel that physical well-being that you get out of it, right? And that's not something you think that much about, how big an impact it has. That I must say. So I definitely intend to keep doing it. [Participant 3, female] When I'm in good physical shape, I recover easier after the attacks that I feel that I have, right? [Participant I0, male] The exercise program kicked me over to the other side again, a couple of months ago I looked myself deep in the eyes and told myself you are only 61 there are plenty of good years in you. [Participant I, male] I got confirmed in my... in the way I handled it ...that maybe it is the way it should be handled... [Participant 7, female] Interviewer: "it was a flu?" [Participant 7, female]

Patient: "Yes I think it made my heart jumpy" [Participant 7, female]

So I have to learn to live with the limitations I have today. [Participant 6, male]

I think as a parent then we try to hide that we are not feeling well... in relation to the kids, right. [Participant 7, female] So, I am sometimes aware of it like... Walking around and feeling like, "Is it there now" and "what now?"... and feeling if it is there all the time. [Participant 9, male]

But I don't know if the atrial fibrillation progress in one way or another have or actually I know in some way that the atrial fibrillation has substantiated some of my fear and anxiety and trembling. [Participant I, male]

You also get a little struck on the psychological aspect I think. You can't do the things that you want to do. It has been extremely difficult for me. [ Participant 6, male]

Well if this [pointing to his heart] does not work then there is not that much to do if I get in that situation, even though early in the process I was told that it is not life threatening, that it wasn't dangerous unless you do not get the right treatment then it affects you anyway. [Participant I, male]

A need for support to move on I just think that the psychological aspect came sneaking along the way, right? [Participant 6, male]

I don't talk much about it, so... In that way you can say that I have not talked to anybody about it... [Participant 8, male] Yes my wife she wanted to know what we had talked about: "what did you actually talk about?" oh yes and if I thought I could drop dead, right? That was the essence of it and how it affected my everyday life. [Participant 10, male] But he [her husband] doesn't want it. So I don't want to use the energy to tell him a lot. I'm not very good at that. Because I don't want to listen to all those stupid questions, or they are not stupid, right? But I can't solve them, right? [Participant 3, female]

Regain confidence in own mental strength by support from professionals And some of the psychological things, I have talked to the nurse about and she thinks that it is a good way to do it... so it is actually nice in that way to be confirmed in that you handle the situation the right way. [Participant 8 , male] Otherwise I chose to come in there, right? Even though I had a long way in here I thought it was very nice to come here. Also because you were all of a sudden told that you were not totally stupid [laughs]. Well she could tell you that when you are saying this and this, other patients tell her the same and then you think oh, ok then maybe it is not just something I make up. [Participant 6 , male]

The feeling of being understood is what I think is very important. Because I can sit and talk to my friends all day but they can only look at me, you cannot look at me and see that I'm feeling bad, since I have not broken an arm, well... so getting understood and so on. That was very good for me. [Participant 6, male]

Normally it is my daughter that asked me for advice, right. And then all of a sudden you stand there not knowing how to tackle the situation, right? And then it was good to be able to talk to and get advice [from the nurse]. [Participant 7, female]

Note: The responses were translated from Danish to English by the first author, Signe S Risom.

help if they got heart palpitations or other symptoms. Some described how they purposely performed the physical exercises next to the defibrillator in case they needed it or how they felt safe training wearing T-shirts with wireless integrated electrocardiogram electrodes that were offered as a part of the program. Since participants did not experience physical symptoms while training, they slowly regained their belief in body strength:

But because I haven't had any trouble of that kind, I toned down that fear of collapsing [...] It has been toned down while in the program [...]. [Participant 10, male]

By participating in training sessions together with other participants, their feelings of belonging to a team where mutual support was experienced strengthened.
[...] we all have the same background, although it is different things we are treated for, all of it is the same, it is our heart it has been about and that is why you get that feeling of belonging to a team, I felt that at least - and you asked each other what have you been treated for and how are you doing now. [Participant 1, male]

\section{Still struggling with fear of AF symptoms}

Some participants experienced AF symptoms and some did not; however, all of them spoke about the fear of AF recurring and being hyperaware of body symptoms, and they expressed how they discussed that with the nurse. Participants were very afraid of AF symptoms returning and this fear influenced the way they lived their lives: 
I can feel my pulse increase in a way I have never before felt and then immediately I react like this: oh no is that an AF attack coming, right? [Participant 1, male]

Most participants had experienced symptoms like low sleep quality, tiredness, headache, and tightness of the chest that they interpreted as AF or stress symptoms. Some participants described how they normally understand everything in life from a rational point of view. Now they were feeling stressed and hyperaware of their body signals and since that could not be described rationally it made them frustrated. Some described how their biggest fear was to get a stroke and end up in a wheelchair.

Also... It is weird, my biggest fear was not to die, my biggest fear was to end up in a wheelchair. [Participant 4, male]

That's it... I'm not afraid of something with the heart.

Something that I am afraid of, if there is something that I am afraid of, is that one got a stroke or a thrombosis or something like that, then you end up like a vegetable... Something like that I'm afraid of... [Participant 8, male]

During psychoeducational sessions with the nurse, participants expressed the need to talk about the fear of AF returning and other health concerns.

I think the feeling of being understood is really important. I could talk to my friends about it, but they can only look at me, and see that I am uncomfortable, they cannot see any physical changes. So, the feeling of being understood was really good for me. [Participant 6 , male]

Participants reported that their largest challenge after the ablation was that they mentally were not back to their old selves.

So physiologically - if you look in the records - it seems to be going really well with me ... psychologically it's just not that exciting. [Participant 8, male]

Participants found psychological problems demoralizing and some even reported fear of death.

[...] it made me reflect on why the hell are you reacting on this? So, death anxiety, what is that for example? [Participant 10 , male]

Mentally, it is just not that exciting. That is where it is - is crazy - because ... I cannot even make any logical explanations about it myself, but it's just like ... you do not have that ... self-esteem I will not say is the right word but ... but you do not feel that you are completely okay... [Participant 1, male]

\section{A need for support to move on}

It was not enough for participants to talk to family members or friends about their worries because they found that they did not understand them and neither could they help. The participants highlighted the need to talk to a health professional. The nurse consultations offered a situation where it was acceptable to discuss specific questions or concerns about AF.

With regard to the psychological aspects, the participant's families were either a support or did not interfere with the healing process. Some participants felt that they needed to protect themselves and their spouse by not sharing that they did not feel good mentally.

\section{$[\ldots]$ and I have probably not told my husband that much $[\ldots]$}

And the same patient later said:

So I do not share that much with him, because then he begins ask more questions. And then I say: stop, I do not want to talk about it... so it is self-inflicted you can say.

[Participant 3, female]

Some families were not involved while the participants were a part of the rehabilitation program whereas others were worried and expressed interest in the program and recovering process. Some participants described how the consultations were a basis for conversations with spouse and children about the participant's feelings:

It is the wife but also the big boys that are worried and interested.

Participants experienced different levels of how their disease influenced their families. Most often both their children and spouses were affected.

\section{Regaining confidence in one's own mental strength with support from professionals} Many of the participants described how they felt it was positive to participate in the psychoeducational consultations. They felt that they were taken seriously and that their feelings and thoughts were validated during the consultations. They reported that the consultations introduced tools to handle everyday life and to handle the fear that AF symptoms or the thought that symptoms may appear often caused:

It made me reflect about my reaction to this. Fear of death, what is it? We talked about that and how I could avoid it. [Participant 10, male]

The participants expressed that it felt good and safe that it was the same nurse for all the consultations, the opposite of what they often experienced in the health care system: 
[...] Most of the time, all I need is a consistent course with someone who can guide me in the things that I am not very good at dealing with [...]. [Participant 1, male]

They described that they had discussed fear of death and had received tools to live with the fear and moved on. Some expressed that they through the consultations realized that they were stronger than they thought they were.

[...] I think it was our conversations as such, oh, that made me realize that I was down and made me realize that I was stronger than I thought. Our conversations and reflections helped me to move on. [Participant 1, male]

On the other hand, others described how they had discussed fundamental issues that they were dealing with and how the consultations had opened up issues that were bigger than they had time to discuss in the four consultations they had as a part of the trial.

[...] So I am sitting and talking to a nurse who I personally feel very comfortable with and so on and who is listening, but in reality the conversations with her opens up for some things that I actually needed to talk more about. [Participant 1 , male]

\section{Discussion}

Biobehavioral outcomes of programs designed to improve physical fitness and the cardiovascular risk factor profile of patients diagnosed with AF have been reported, ${ }^{8,15}$ but how patients describe their experience in participating in these programs is lacking. This study provides an insight into patients' rehabilitation experiences that can be used to guide further development of rehabilitation programs for patients with AF.

\section{Strengthening belief in one's own physical capacity and survival}

Participants in this study expressed that the exercise training sessions increased their self-efficacy for exercise and boosted their motivation to pursue their goals. The findings here are consistent with Bandura's social cognitive theory ${ }^{16}$ that proposes that self-efficacy is promoted by the achievement of small goals and provides a foundation for accomplishing more challenging ones. In the interviews, participants' success in achieving small goals led to the belief that they could regain their stamina and cardiovascular fitness. Perceptions such as these were reported by participants of other rehabilitation programs for cardiovascular conditions ${ }^{17-20}$ and patients with implantable cardioverter defibrillators (ICDs). ${ }^{21-23}$
The importance of social support as motivation to adopt new behaviors, another feature of Bandura's social cognitive theory ${ }^{16}$ was evident in participant responses. The sense of belonging to a group and encouraging one another to achieve goals was expressed by participants in this study as well as those in previous qualitative descriptions of cardiac rehabilitation experiences. ${ }^{17,22,23}$ Participants reported that having the physical therapist present gave them a sense of security that allowed them to overcome fear and anxiety and push themselves to higher levels which, in turn, increased their self-efficacy. These findings are similar to those of ICD participants ${ }^{22,23}$ who like AF participants feared that exercise could trigger an arrhythmia.

\section{Still struggling with fear of AF symptoms}

After ablation, participants continued to be hypervigilant for symptoms, constantly scanning their bodies for any evidence that AF had returned. They had not anticipated the mental/ emotional stress and fatigue that persisted in the weeks after the ablation procedure. Some understood that their fears were irrational, but were unable to cognitively reframe them. Similar hypervigilant responses have been reported in other qualitative studies, but participants in those studies had not yet undergone ablation. ${ }^{5,24}$ In contrast to our findings, Withers et al reported that some patients undergoing ablation for a variety of cardiac arrhythmias experienced a rapid improvement in psychological well-being following ablation. However, the authors did not specify whether participants with AF reported those early benefits. ${ }^{25}$ One quantitative study revealed that anxiety and depression decreased from baseline to 12 months after ablation treatment for paroxysmal $\mathrm{AF}^{26}$ Other than what we have documented in this study, little is known about participants' psychological well-being earlier in recovery after the AF ablation procedure.

\section{A need for support to move on}

Participants in this study expressed reluctance to share aspects of AF-related psychological distress with family members, but were able to confide their fears and worries to the nurse in the rehabilitation program. Quantitative reports document that AF adversely affects spouses/partners' quality of life and psychological well-being. ${ }^{27,28} \mathrm{McCabe}$ documented that $57 \%$ of participants reported that $\mathrm{AF}$ produced negative consequences for family members. ${ }^{29}$ However, few reports describe how patients with AF share with family, friends, and coworkers the effect AF has on their own psychological well-being. Consistent with findings in this study, some participants of qualitative studies have reported not wanting to 
burden family members by expressing feelings of depression or worry. ${ }^{24,25}$ Others reported feeling unsupported, emotionally isolated, and misunderstood by family members and friends who could not grasp the effect of symptoms on their ability to function. ${ }^{5,25}$ The findings demonstrate the need to generate deeper knowledge of how living with AF affects the psychological dynamics of family/partner relationships.

\section{Regaining confidence in one's own mental strength by support from professionals}

The interventions used in the psychoeducational sessions of this rehabilitation program were guided by Parse's Human Becoming Practice Methodologies, ${ }^{13}$ a feature of this study that was reported in only two other previous qualitative studies that examined participants' rehabilitation experiences..$^{23,30}$ Previous studies reported that patients with AF felt their concerns were dismissed and undervalued by clinicians and did not receive guidance to help them cope with the emotional challenges of living with $\mathrm{AF}^{5,25}$ By contrast, in this study, with the support of the nurse, patients felt comfortable enough to discuss their fears, the meaning living with AF had for them, were able to look forward to achieving new goals, and see themselves as making progress toward those goals. Our findings are similar to Berg et al who conducted a rehabilitation trial including ICD patients. ${ }^{23}$

In this study and others, ${ }^{22,23,31}$ the support of clinicians was important for coping with the fear and anxiety of recurrent arrhythmias. The ability to face fears of triggering an arrhythmia is particularly important in patients with AF because managing anxiety and fear effectively is important for avoiding adrenergic stimulation that may trigger an arrhythmia. ${ }^{32}$ The therapeutic benefit of a consistent provider of the psychoeducational sessions was reported in this study, but not mentioned in other qualitative evaluations of cardiac rehabilitation programs. ${ }^{17,22}$

\section{Limitations}

Some participants were interviewed right after the rehabilitation intervention ended and some were interviewed 1-2 months after. Recollection of events that occurred weeks to months before the interviews may have been recalled differently. The participants were recruited as part of the CopenHeartRFA trial, which may have influenced the general representativeness of patients undergoing ablation for AF. Although the interviewer had no contact with the participants prior to the interviews, the participants knew the interviewer as an employee of the hospital where treatment was received and that the interviewer was a coinvestigator in the study. It is possible that participants' responses may have been biased in favor of wanting to please the interviewer; participants may have been reluctant to express any negative experience associated with the study intervention.

\section{Conclusion}

The four themes describe the participants' perceptions of the intervention's positive effect on promoting self-efficacy for engaging in physical activity and the benefit of a safe environment for processing the emotional responses during the recovery period. The data clearly reveal the value of the therapeutic relationship between the patient and the rehabilitation provider (both therapist and nurse) for reducing insecurities and validating emotional responses. Indeed, our results show that in the early post-ablation period, participants were particularly vulnerable to fear and anxiety and benefited from psychoeducational consultations to cope with AF-related stressors. The results suggest that patients after ablation for AF would benefit from rehabilitation including both a physical exercise component and psychoeducational consultations focusing on fear and anxiety. However, additional studies that evaluate patients' perspectives are warranted to determine whether the benefits of participating in the CopenHeartRFA program can be reproduced in other settings.

\section{Acknowledgments}

Thanks to the patients who participated in the CopenHeart trial and especially to the 10 patients who contributed to these interviews. Thanks to Anne Alexandrine Øhlers for assisting with transcribing the interviews and technical help with setting up the manuscript. We would also like to thank Iman Hassan Abdullahi for helping in the analysis process and Signe Westh Christensen for assisting with the layout of the article and finally the entire CopenHeart team for designing and conducting the CopenHeartRFA trial.

This study was supported by the Danish Strategic Research Council (grant number 10-092790); The Heart Centre, Rigshospitalet; Metropolitan University College; The Lundbeck Foundation (grant number FP 62/2011FP, 74/2012, 2014-3962); and the Health Foundation (grant number 15-B-0114), Denmark. The abstract of this paper was presented at the EuroHeartCare 2017 in Jonkoping as a poster presentation with interim findings.

\section{Disclosure}

The authors report no conflicts of interest in this work. 


\section{References}

1. Chugh SS, Havmoeller R, Narayanan K, et al. Worldwide epidemiology of atrial fibrillation: a global burden of disease 2010 study. Circulation. 2014;129(8):837-847.

2. Naccarelli GV, Varker H, Lin J, Schulman KL. Increasing prevalence of atrial fibrillation and flutter in the United States. Am J Cardiol. 2009;104(11):1534-1539.

3. Kirchhof P, Benussi S, Kotecha D, et al. 2016 ESC guidelines for the management of atrial fibrillation developed in collaboration with EACTS: the Task Force for the management of atrial fibrillation of the European Society of Cardiology (ESC). Eur Heart J. 2016;18(11): 1609-1678.

4. January CT, Wann FLS, Joseph F, et al. 2014 AHA/ACC/HRS guideline for the management of patients with atrial fibrillation: executive summary. J Am Coll Cardiol. 2014;64(21):2246-2280.

5. McCabe PJ, Schumacher K, Barnason SA. Living with atrial fibrillation: a qualitative study. J Cardiovasc Nurs. 2011;26(4):336-344.

6. Anderson L, Taylor RS. Cardiac rehabilitation for people with heart disease: an overview of Cochrane systematic reviews. Cochrane Database Syst Rev. 2014;12:CD011273.

7. Risom SS, Zwisler AD, Rasmussen TB, et al. The effect of integrated cardiac rehabilitation versus treatment as usual for atrial fibrillation patients treated with ablation: the randomised CopenHeartRFA trial protocol. BMJ Open. 2013;3(2):2012-2377.

8. Risom SS, Zwisler A-D, Rasmussen TB, et al. Cardiac rehabilitation versus usual care for patients treated with catheter ablation for atrial fibrillation: results of the randomized CopenHeartRFA trial. Am Heart J. 2016;181:120-129.

9. Creswell JW, Clark VLP. Designing and Conducting Mixed Methods Research. Thousand Oaks, CA: SAGE Publications; 2007.

10. Kvale S, Brinkmann S. Interviews: Learning the Craft of Qualitative Research Interviewing. 2nd ed. Thousand Oaks, CA: Sage Publications; 2009.

11. Morse J. Drowning in data. Qual Health Care Res. 1993;3:267-269.

12. Moore SM. Women's views of cardiac rehabilitation programs. J Cardiopulm Rehabil. 1996;16(2):123-129.

13. Parse RR. The Human Becoming School of Thought: A Perspective for Nurses and Other Health Professionals. Thousand Oaks, CA: Sage; 1998.

14. Crabtree B, Miller W, editors. Doing Qualitative Research. Thousand Oaks, CA: Sage Publications; 1999:163-178.

15. Pathak RK, Middeldorp ME, Lau DH, et al. Aggressive risk factor reduction study for atrial fibrillation and implications for the outcome of ablation: the ARREST-AF cohort study. J Am Coll Cardiol. 2014;64(21): 2222-2231.

16. Glanz K, Bishop DB. The role of behavioral science theory in development and implementation of public health interventions. Annu Rev Public Health. 2010;31:399-418.

17. Simony CP, Pedersen BD, Dreyer P, Birkelund R. Dealing with existential anxiety in exercise-based cardiac rehabilitation: a phenomenological-hermeneutic study of patients' lived experiences. J Clin Nurs. 2015;24(17-18):2581-2590.
18. Leahy RA, Davenport EE. Home monitoring for cardiovascular implantable electronic devices. AACN Adv Crit Care. 2015;26(4):343-355.

19. Rodiere M. What is the true benefit of thrombectomy devices in acute upper and lower limb DVT? Cardiovasc Intervent Radiol. 2013;36: S183-S184.

20. Devi R, Carpenter C, Powell J, Singh S. Exploring the experience of using a web-based cardiac rehabilitation programme in a primary care angina population: a qualitative study. Int J Ther Rehabil. 2014;21(9): 434-440.

21. Berg SK, Moons P, Christensen AV, Zwisler A-D, Pedersen BD, Pedersen PU. Clinical effects and implications of cardiac rehabilitation for implantable cardioverter defibrillator patients: a mixed-methods approach embedding data from the Copenhagen outpatient programmeimplantable cardioverter defibrillator randomized clinical trial with qualitative data. J Cardiovasc Nurs. 2015;30(5):420-427.

22. Morken IM, Norekvål TM, Isaksen K, Munk PS, Karlsen B, Larsen AI. Increased confidence to engage in physical exertion: older ICD recipients' experiences of participating in an exercise training programme. Eur J Cardiovasc Nurs. 2013;12(3):261-268.

23. Berg SK, Pedersen BD, Svendsen JH, Zwisler AD, Kristensen L, Pedersen PU. COPE-ICD: patient experience of participation in an ICD specific rehabilitation programme. Eur J Cardiovasc Nurs. 2012;11(2): 207-215.

24. Ekblad H, Rönning H, Fridlund B, Malm D. Patients' well-being: experience and actions in their preventing and handling of atrial fibrillation. Eur J Cardiovasc Nurs. 2013;12(2):132-139.

25. Withers KL, Wood KA, Carolan-Rees G, Patrick H, Lencioni M, Griffith M. Living on a knife edge - the daily struggle of coping with symptomatic cardiac arrhythmias. Health Qual Life Outcomes. 2015;13:86.

26. Sang C-H, Chen K, Pang X-F, et al. Depression, anxiety, and quality of life after catheter ablation in patients with paroxysmal atrial fibrillation. Clin Cardiol. 2013;36(1):40-45.

27. Bohnen M, Shea JB, Michaud GF, et al. Quality of life with atrial fibrillation: do the spouses suffer as much as the patients? Pacing Clin Electrophysiol. 2011;34(7):804-809.

28. Dalteg T, Benzein E, Sandgren A, Malm D, Arestedt K. Associations of emotional distress and perceived health in persons with atrial fibrillation and their partners using the actor-partner interdependence model. J Fam Nurs. 2016;22(3):368-391.

29. McCabe PJ. Predictors of Symptoms and Psychological Distress in Patients with Recurrent Symptomatic Atrial Fibrillation [dissertation]. Omaha: University of Nebraska Medical Center; 2009.

30. Hansen TB, Zwisler AD, Berg SK, Sibilitz KL, Buus N, Lee A. Cardiac rehabilitation patients' perspectives on the recovery following heart valve surgery: a narrative analysis. JAdv Nurs. 2016;72(5):1097-1108.

31. Qintar M, George JJ, Panko M, et al. A prospective study of anxiety in ICD patients with a pilot randomized controlled trial of cognitive behavioral therapy for patients with moderate to severe anxiety. J Interv Card Electrophysiol. 2015;43(1):65-75.

32. Lampert R, Jamner L, Burg M, et al. Triggering of symptomatic atrial fibrillation by negative emotion. J Am Coll Cardiol. 2014;64(14): 1533-1534.
Journal of Multidisciplinary Healthcare

\section{Publish your work in this journal}

The Journal of Multidisciplinary Healthcare is an international, peerreviewed open-access journal that aims to represent and publish research in healthcare areas delivered by practitioners of different disciplines. This includes studies and reviews conducted by multidisciplinary teams as well as research which evaluates the results or conduct of such teams or health

\section{Dovepress}

care processes in general. The journal covers a very wide range of areas and welcomes submissions from practitioners at all levels, from all over the world. The manuscript management system is completely online and includes a very quick and fair peer-review system. Visit http://www.dovepress.com/ testimonials.php to read real quotes from published authors. 